\title{
Low-flow support of the chronic pressure-overloaded right ventricle induces reversed remodeling
}

\author{
Tom Verbelen, MD, ${ }^{\mathrm{a}, \mathrm{b}}$ Piet Claus, MSc, PhD, ${ }^{\mathrm{c}}$ Daniel Burkhoff, MD, PhD, ${ }^{\mathrm{d}}$ \\ Ronald B. Driesen, MSc, PhD, ${ }^{e}$ Chandan Kadur Nagaraju, MSc, ${ }^{e}$ \\ Erik Verbeken, MD, PhD, ${ }^{f}$ Karin Sipido, MD, PhD, ${ }^{e}$ Marion Delcroix, MD, PhD, ${ }^{g, h}$ \\ Filip Rega, MD, PhD, ${ }^{a, b}$ and Bart Meyns, MD, PhD ${ }^{a, b}$
}

\begin{abstract}
From the ${ }^{a}$ Department of Cardiac Surgery, University Hospitals Leuven, Leuven, Belgium; ${ }^{b}$ Division of Experimental Cardiac Surgery, Department of Cardiovascular Sciences, University of Leuven, Leuven, Belgium; ${ }^{c}$ Division of Cardiovascular Imaging and Dynamics, Department of Cardiovascular Sciences, University of Leuven, Leuven, Belgium; ${ }^{d}$ Division of Cardiology, Columbia University College of Physicians and Surgeons, New York, New York, USA; ${ }^{e}$ Division of Experimental Cardiology, Department of Cardiovascular Sciences, University of Leuven, Leuven, Belgium; ${ }^{f}$ Division of Translational Cell \& Tissue Research, Department of Imaging \& Pathology, University of Leuven, Leuven, Belgium; ${ }^{g}$ Respiratory Division, University Hospitals Leuven Leuven, Belgium; and the ${ }^{h}$ Department of Clinical and Experimental Medicine, University of Leuven, Leuven, Belgium.
\end{abstract}

\section{KEYWORDS: \\ Pulmonary arterial hypertension; right ventricular failure; pressure-overloaded right ventricle; right ventricular mechanical support}

\begin{abstract}
BACKGROUND: Mechanical right ventricular (RV) support in pulmonary arterial hypertension patients has been feared to cause pulmonary hemorrhage and to be detrimental for the after-load-sensitive RV. Continuous low-flow pumps offer promise but remain insufficiently tested.

METHODS: The pulmonary artery was banded in 20 sheep in this study. Eight weeks later, a Synergy micro-pump (HeartWare International, Framingham MA) was inserted in 10 animals, driving blood from the right atrium to the pulmonary artery. After magnetic resonance imaging, hemodynamics and RV pressure-volume loop data were recorded. Eight weeks later, RV function was assessed in the same way, followed by histologic analysis of the ventricular tissue.

RESULTS: During the 8 weeks of support, RV volumes and central venous pressure decreased significantly, whereas RV contractility increased. Pulmonary artery pressure increased modestly, particularly its diastolic component. RV contribution to total right-sided cardiac output increased from $12 \pm 12 \%$ to $41 \pm 9 \%\left(p<1 \times 10^{-4}\right)$. After pump inactivation, and compared with 8 weeks earlier, RV volumes had significantly decreased, tricuspid valve regurgitation had almost disappeared, and RV contractility had significantly increased, resulting in significantly increased RV forward power $(0.25 \pm$ 0.05 vs $0.16 \pm 0.06 \mathrm{~W}, p=0.014$ ). Fulton index and RV myocyte size were significantly smaller, and without changes in fibrosis, when compared with controls.

CONCLUSIONS: Prolonged continuous low-flow RV mechanical support significantly unloads the chronic pressure-overloaded RV and improves cardiac output. After 8 weeks, RV hemodynamic recovery and reverse remodeling begin to occur, without increased fibrosis.

J Heart Lung Transplant mun; :m:m-nu
\end{abstract}

(C) 2017 International Society for Heart and Lung Transplantation. All rights reserved.
Reprint requests: Tom Verbelen, MD, Department of Cardiac Surgery, University Hospitals Leuven, Herestraat 49, 3000 Leuven, Belgium. Telephone: +3216344260. Fax: +3216344616.

E-mail address: tom.verbelen@kuleuven.be
Pulmonary arterial hypertension (PAH) is caused by a pathologic increase in pulmonary vascular resistance (PVR) and leads to right ventricular (RV) failure. ${ }^{1}$ Even with modern therapy, mortality is still $15 \%$ within 1 year and 
median survival remains limited to 5 to 6 years, with insufficient functional improvement in many survivors. ${ }^{2}$ Bilateral lung transplantation (bLTx) remains the only curative option. Unfortunately, most patients are unlikely to survive until transplantation.

PAH-associated RV failure is potentially reversible as RV function generally recovers after bLTx. ${ }^{3}$ Therefore, surgical strategies to increase cardiac output (CO) and reduce RV wall stress should be elaborated. Atrial septostomy for PAH unloads the RV and improves cardiac index, but procedural mortality approaches $16 \%$. $^{4}$ Right ventricular assist devices (RVADs) could offer the same advantages, but would actively support the RV without arterial oxygen desaturation. Pulsatile device use often results in pulmonary hemorrhage and death. ${ }^{5}$ Continuousflow devices have been successful for acute pressureoverloaded RVs. ${ }^{6}$ However, such devices, with relatively high flow rates, may also result in increased pulmonary pressures and lung injury. ${ }^{7}$ Smaller devices with lower flow capacities may therefore be more appropriate. We recently demonstrated the feasibility and advantages of mechanically supporting acute and chronic pressure overloaded RVs with low flows. ${ }^{8,9}$ Our next goal is to assess the effects of prolonged low-flow RVAD support on chronic pressureoverloaded RVs.

\section{Methods}

\section{Animal preparation}

This study was approved by the animal ethics committee of KU Leuven (P127/2011). The female sheep (Swifter-Charolais) used for the study received humane care in compliance with the "Principles of Laboratory Animal Care" (National Society for Medical Research) and the Guide for Care and Use of Laboratory Animals (Institute of Laboratory Animal Resources, National Institutes of Health). Twenty animals were included (weight $47.3 \pm$ $4.8 \mathrm{~kg}$ and age $10.4 \pm 0.8$ months). After sedation with intramuscular ketamine $15 \mathrm{mg} / \mathrm{kg}$, anesthesia was induced and maintained with isoflurane ( $2 \%$ to $3 \%)$. A volume-controlled respirator (Dräger Cicero; Lübeck, Germany) was used for ventilation. Buprenorphine hydrochloride $0.3 \mathrm{mg}$ and meloxicam $0.5 \mathrm{mg} / \mathrm{kg}$ intravenously were used for analgesia.

\section{Pulmonary artery banding}

Arterial blood pressure (ABP) and central venous pressure (CVP) were measured in an ear artery and a jugular vein, respectively. A Swan-Ganz catheter measured distal pulmonary artery (PA) pressure. After left thoracotomy, a band around the distal pulmonary trunk was tightened as much as hemodynamically tolerated; that is, the ends of the band were fixed together at their end by a clip, resulting in a drop in ABP to a new stable level. Additional clips were placed to increasingly tighten the band around the PA. A new, lower ABP was achieved with each clip. Once the ABP showed continued decline without signs of stabilization, the last clip was removed. A flowmeter (TS420; Transonic Systems Europe BV, Maastricht, The Netherlands) around the proximal PA measured right ventricular cardiac output (RVCO). Pressure lines in the left atrium, just proximal and distal to the band, served to measure corresponding pressures.

\section{Intervention at 8 weeks}

Animals were scanned with 3.0-Tesla magnetic resonance imaging (MRI) (Magnetom, Trio Tim; Siemens Medical Solutions, Erlangen, Germany), with a phased-array body coil over the heart. Cine images were acquired with electrocardiogram gating during suspended respiration, in vertical and horizontal long- and shortaxis planes.

In addition to PA-banding instrumentation, an 18-mm balloon (Reliant; Medtronic, Minneapolis, MN) was inserted in the left jugular vein and then docked in the inferior caval vein 1 day later. In 10 sheep, a Synergy micro-pump (HeartWare International, Framingham, MA) was inserted to withdraw blood from right atrium to the PA. A flowmeter (Sono TT; Emtec, Gennevilliers, France) measured pump flow. A 7F combined pressure conductance catheter, connected to a Sigma M signal processor (both from CD Leycom, Zoetermeer, The Netherlands), was positioned through a stab wound just below the pulmonary valve toward the apex (Figure 1). Pressure, flow and pressure-volume (PV) loop data were recorded for all animals, just before mechanical support initiation, and then 15 minutes after pump activation (22,000 rpm) in 10 animals. PV loops with pre-load reduction were obtained by balloon inflation, but only in the non-supported condition (pre-load reduction during support generated unusable PV loops).

\section{Evaluation at 16 weeks}

Eight weeks later, hemodynamics and PV loop parameters were recorded. MRI was performed on the following day. In RVAD animals, pumps were deactivated and their subcutaneous metal housings were removed 15 minutes before MRI. Subsequently, animals were euthanized using $20 \mathrm{ml} \mathrm{KCl}$ (14.9\%) intravenously after reassurance of adequate anesthesia. During autopsy, the Fulton index [RV / (left ventricle (LV) + septum) weight] was calculated.

\section{Histology}

RV and LV free wall mid-ventricular samples were fixed in $4 \%$ paraformaldehyde in phosphate-buffered saline and processed and

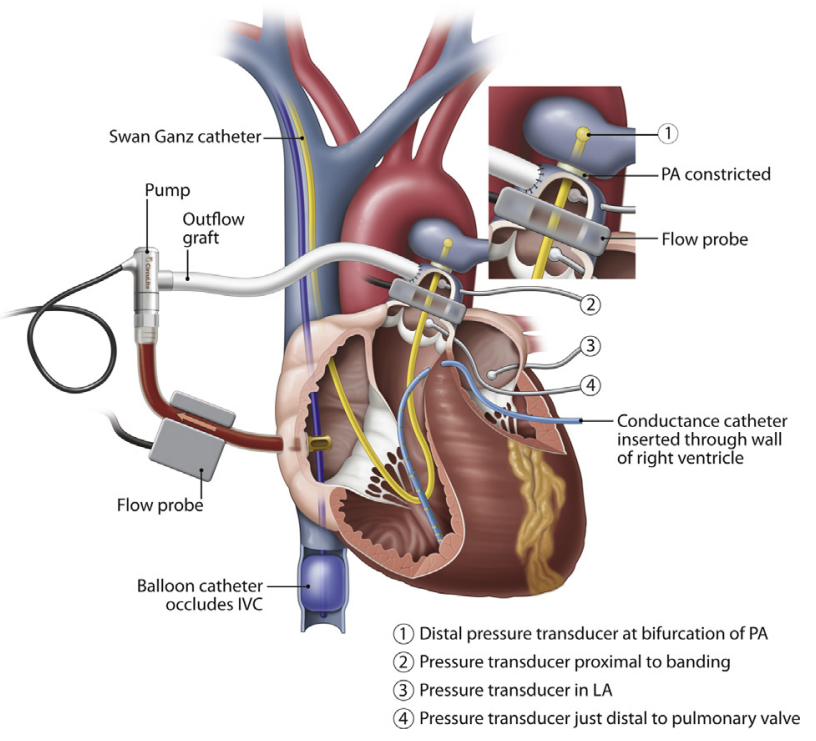

Figure 1 Schematic overview of the experimental set-up. PA = pulmonary artery; LA = left atrium; IVC = inferior vena cava. 
embedded in paraffin. Myocyte hypertrophy was measured in hematoxylin-and-eosin-stained sections, using the mean linear intercept method. ${ }^{10,11}$ In brief, myocytes transected by a probe with a length of $500 \mu \mathrm{m}$ at $200 \times$ magnification were counted on 10 fields per heart and averaged. This number correlates with the inverse of myocyte volumes and indexes myocyte hypertrophy. For fibrotic analysis, $8-\mu$ m-thick paraffin sections were cut and dried. Sections were deparaffinized and rehydrated. Total collagen was visualized using a PicroSirius red staining kit (PolySciences, Bergstrasse, Germany). Sections were then mounted in distyrene plasticizer xylene mounting medium. Images were acquired using a Zeiss Axioplan microscope with an Axiocam $\mathrm{HrC}$ camera (both from Carl Zeiss, Jena, Germany). Polarization microscopy was performed on Sirius red-stained sections to visualize collagen types I and III based on the birefringence properties of collagen. Total amount of fibrosis and collagen types I and III were quantified using AXIOVISION (Zeiss) software by 2 blinded operators.

\section{Data analysis}

Using MATLAB R2013a (The Mathworks, Natick, MA) software, a single, blinded operator delineated on MRI-derived images LV and RV systolic and diastolic inner borders and calculated the corresponding volumes, stroke volume (SV) and CO. Tricuspid valve regurgitation $(\mathrm{TVR})=(\mathrm{RVSV}-\mathrm{LVSV}) / \mathrm{RVSV} \times 100 \%$. As TVR is aggravated to the detriment of forward RVSV, the calculated SV and ejection fraction (EF) obtained from RV endsystolic volume (ESV) and RV end-diastolic volume (EDV) can be misleading and may overestimate the real systolic RV function. Forward RVSV corresponds to LVSV, thus forward RVEF = $(\mathrm{LVSV} / \mathrm{RVEDV} \times 100)$; total $\mathrm{CO}=\mathrm{RVCO}+$ pump flow; RV power $=$ mean proximal $\mathrm{PA}-$ pressure $\times \mathrm{RVCO} \times 0.0022$; and total power $=$ mean $\mathrm{PA}$ pressure just before band $\times$ total $\mathrm{CO} \times$ 0.0022 .

PV loops were analyzed using CD Leycom and MATLAB software. The end-systolic pressure-volume relationship (ESPVR) was determined by pre-load reduction (inactive pump) or by singlebeat estimation (active pump) by means of sine curve fitting [A $\times$ $\sin (\mathrm{Bt}+\mathrm{C})+\mathrm{D}]$ and the Levenberg-Marquardt procedure. ${ }^{12,13}$ From this, ESPVR slope ( $\mathrm{E}_{\mathrm{es}}$, end-systolic elastance) and volume axis intercept $\left(\mathrm{V}_{0}\right)$ were determined. ESPVR $\mathrm{V}_{40}$ (volume at endsystolic pressure [ESP] of $40 \mathrm{~mm} \mathrm{Hg}$ ) was calculated and served as additional index of contractility. ${ }^{14}$ The end-diastolic pressurevolume relationship (EDPVR) was determined using pre-load reduction (inactive pump) or computational single-beat (active pump) methods. ${ }^{14,15}$ From these curves $\left(\mathrm{P}=\alpha \mathrm{V}^{\beta}\right)$, EDPVR $\mathrm{V}_{30}$ (volume at end-diastolic pressure [EDP] of $30 \mathrm{~mm} \mathrm{Hg}$ ) was calculated as index of capacitance. To compare these curves,

Table 1 Hemodynamics Before and After Banding the Pulmonary Artery

\begin{tabular}{|c|c|c|c|}
\hline & Pre-banding & Post-banding & $p$-value \\
\hline \multicolumn{4}{|l|}{ Weight (kg) } \\
\hline Pump group $(n=8)$ & $46.9 \pm 4.2$ & & \\
\hline Control group $(n=8)$ & $47.5 \pm 5.1$ & & \\
\hline \multicolumn{4}{|l|}{ Age (months) } \\
\hline Pump group $(n=8)$ & $10.5 \pm 0.6$ & & \\
\hline Control group $(n=8)$ & $10.2 \pm 0.9$ & & \\
\hline \multicolumn{4}{|l|}{$\mathrm{mABP}(\mathrm{mm} \mathrm{Hg})$} \\
\hline Pump group $(n=8)$ & $59.6 \pm 3.4$ & $47.9 \pm 7.1$ & $<1 \times 10^{-5}$ \\
\hline Control group $(n=8)$ & $60.1 \pm 2.8$ & $48.4 \pm 5.9$ & $<1 \times 10^{-4}$ \\
\hline \multicolumn{4}{|c|}{ mPAP proximal band $(\mathrm{mm} \mathrm{Hg})$} \\
\hline Pump group $(n=8)$ & $15.5 \pm 2.9$ & $32.7 \pm 4.7$ & $<1 \times 10^{-4}$ \\
\hline Control group $(n=8)$ & $15.8 \pm 3.2$ & $32.5 \pm 5.1$ & $<1 \times 10^{-7}$ \\
\hline \multicolumn{4}{|l|}{ mPAP distal band $(\mathrm{mm} \mathrm{Hg})$} \\
\hline Pump group $(n=8)$ & $15.5 \pm 2.9$ & $13.6 \pm 3.2$ & $<1 \times 10^{-4}$ \\
\hline Control group $(n=8)$ & $15.8 \pm 3.2$ & $13.9 \pm 3.4$ & $<1 \times 10^{-3}$ \\
\hline \multicolumn{4}{|l|}{ CVP $(\mathrm{mm} \mathrm{Hg})$} \\
\hline Pump group $(n=8)$ & $7.7 \pm 1.7$ & $12.3 \pm 2.0$ & $<1 \times 10^{-3}$ \\
\hline Control group $(n=8)$ & $7.9 \pm 1.9$ & $12.8 \pm 1.8$ & $<1 \times 10^{-5}$ \\
\hline \multicolumn{4}{|l|}{ LAP $(\mathrm{mm} \mathrm{Hg})$} \\
\hline Pump group $(n=8)$ & $4.3 \pm 1.7$ & $5.8 \pm 1.8$ & $<0.01$ \\
\hline Control group $(n=8)$ & $4.4 \pm 1.5$ & $6.1 \pm 1.7$ & $<0.01$ \\
\hline \multicolumn{4}{|l|}{ Heart rate (beats/min) } \\
\hline Pump group $(n=8)$ & $88 \pm 10$ & $87 \pm 11$ & 0.7842 \\
\hline Control group $(n=8)$ & $93 \pm 12$ & $91 \pm 11$ & 0.7253 \\
\hline \multicolumn{4}{|l|}{ CO (liters/min) } \\
\hline Pump group $(n=8)$ & $3.73 \pm 0.75$ & $2.73 \pm 0.78$ & $<1 \times 10^{-6}$ \\
\hline Control group $(n=8)$ & $3.75 \pm 0.66$ & $2.69 \pm 0.68$ & $<1 \times 10^{-7}$ \\
\hline \multicolumn{4}{|c|}{ Resistance across banding ( $\left.\mathrm{dyn} / \mathrm{s} / \mathrm{cm}^{5}\right)$} \\
\hline Pump group $(n=8)$ & & $570 \pm 148$ & \\
\hline Control group $(n=8)$ & & $564 \pm 164$ & \\
\hline
\end{tabular}

Resistance across banding $=80 \times(\mathrm{mPAP}$ proximal band $-\mathrm{mPAP}$ distal band) $/$ CO. No statistically significant differences were found between the pump and control groups. CO, cardiac output; CVP, central venous pressure; LAP, left atrial pressure; mABP, mean arterial blood pressure; mPAP, mean pulmonary arterial pressure. 
curve-fit expressions were linearized using logarithmic transformation (ln) of pressure and volume data, resulting in $\beta$ as slope and $(-\ln (\alpha) / \beta)$ as $\mathrm{V}_{0}{ }^{14}$

Data are presented as mean \pm standard deviation. Statistical analysis was performed using STATISTICA version 10.0 (StatSoft, Inc., Tulsa, OK) software. Wilcoxon's matched pairs test was used for procedure-dependent comparison. Mann-Whitney $U$-test was used for comparisons between 2 experimental groups. $p<$ 0.05 was considered statistically significant.

\section{Results}

PA banding caused comparable hemodynamic changes in both groups (Table 1). All animals developed clinical signs of RV failure. Tachypnea developed from the first postoperative day. Abdominal sonography confirmed the presence of large amounts of ascites. Animals that presented too dyspneic were treated with furosemide and ascites drainage. Compared with the acute effects of PA banding, EDV, ESV, central venous pressure (CVP), SV and RV compliance and capacitance increased significantly, whereas RV peak pressures, ESPs and pulonary artery pressures (PAPs) in PA pressures decreased. Together with an apparently worsened contractility and ventriculo-arterial coupling, these findings indicate deterioration of RV function (Tables 2 to 5). PA banding and pump activation in chronic pressureoverloaded RVs have been described before. ${ }^{8,9}$ The presumed TVR in these studies was thus confirmed by MRI (Table 2).

\section{Drop-outs and hemocompatibility}

Two animals died, 9 and 14 days after pump implantation, 1 due to sepsis. Autopsies revealed fresh and organized clotting in outflow grafts and fresh clotting in pump housings. As pump currency increased, another animal underwent surgical revision 2 weeks after pump implantation. Fresh clotting was successfully removed from the outflow graft and housing, and the pump continued to work properly. One control animal died at 15 weeks and 4 days due to RV failure. Another control collapsed at 16 weeks while initiating anesthesia. Ventricular histology of these 2 animals was included for analysis.

\section{RV function during 8 weeks of mechanical support}

ESP and peak RV pressure increased significantly, whereas CVP, EDV and ESV decreased significantly, resulting in a significantly higher EF, stroke work (SW) and pressurevolume area (PVA). Increased $\mathrm{E}_{\mathrm{es}}$ and significantly decreased $\mathrm{V}_{0}$ indicate increased contractility, confirmed by the significantly decreased $\mathrm{V}_{40}$. RV capacitance decreased significantly (Tables 3 to 5 and Figures 2 and 3A). These findings indicate RV recovery, as manifested by significant increasing RVCO and power. Intrinsic RVCO increased from $12 \pm 12 \%$ to $41 \pm 9 \%$ of total CO $\left(p<1 \times 10^{-4}\right)$. Compared with the unsupported condition just before pump activation 8 weeks earlier, total $\mathrm{CO}$ on support had increased significantly $(p<0.05)$.

\section{RV function after 8 weeks of mechanical support}

Unsupported RV parameters were compared with those from 8 weeks earlier (Tables 2 to 5 and Figures 2 and 3B). MRI showed significantly decreased EDV and ESV with significantly increased EF. Calculated RVCO did not change. However, as TVR disappeared almost completely, forward RVCO increased, as proven by the significantly increased calculated LVCO. In this regard, the SV and EF findings from the PV measurements in Table 4 should not be misinterpreted because they are largely overestimated due to the inclusion of TVR. True forward SV and EF data are given in Table 2 and both increased significantly after 8 weeks of support. Peak RV pressure, ESP and PA

Table 2 MRI-derived Parameters

\begin{tabular}{|c|c|c|c|c|}
\hline & \multicolumn{2}{|c|}{ Pump group $(n=8)$} & \multicolumn{2}{|c|}{ Control group $(n=8)$} \\
\hline & 8 weeks & 16 weeks & 8 weeks & 16 weeks \\
\hline TVR $(\%)$ & $24 \pm 14$ & $5 \pm 5^{a}$ & $20 \pm 17$ & $42 \pm 21^{a, b}$ \\
\hline HR (beats/min) & $90 \pm 16$ & $98 \pm 21$ & $101 \pm 16$ & $95 \pm 17$ \\
\hline LVCO (liters/min) & $2.45 \pm 0.74$ & $3.47 \pm 1.01^{a}$ & $2.87 \pm 0.40$ & $2.78 \pm 1.02$ \\
\hline RVCO (liters/min) & $3.41 \pm 1.61$ & $3.65 \pm 1.01$ & $3.80 \pm 1.15$ & $5.02 \pm 1.63^{a}$ \\
\hline RVEDV $(\mathrm{ml})$ & $130 \pm 29$ & $104 \pm 20^{a}$ & $139 \pm 16$ & $146 \pm 23^{b}$ \\
\hline RVESV (ml) & $92 \pm 26$ & $66 \pm 18^{a}$ & $100 \pm 20$ & $92 \pm 24^{b}$ \\
\hline RVSV (ml) & $38 \pm 12$ & $38 \pm 10$ & $39 \pm 15$ & $54 \pm 18^{a, b}$ \\
\hline RVEF (\%) & $30 \pm 9$ & $37 \pm 10^{a}$ & $28 \pm 11$ & $37 \pm 11^{a}$ \\
\hline fRVEF (\%) & $22 \pm 10$ & $35 \pm 11^{a}$ & $21 \pm 5$ & $21 \pm 7^{b}$ \\
\hline LVEDV (ml) & $67 \pm 7$ & $68 \pm 16$ & $69 \pm 6$ & $68 \pm 10$ \\
\hline LVESV (ml) & $40 \pm 7$ & $32 \pm 8^{a}$ & $40 \pm 5$ & $38 \pm 5$ \\
\hline $\mathrm{LV}(=\mathrm{fRV}) \mathrm{SV}(\mathrm{ml})$ & $28 \pm 7$ & $36 \pm 10^{a}$ & $29 \pm 6$ & $30 \pm 10$ \\
\hline $\operatorname{LVEF}(\%)$ & $41 \pm 9$ & $53 \pm 7^{a}$ & $42 \pm 7$ & $43 \pm 10^{b}$ \\
\hline
\end{tabular}

CO, cardiac output; EDV, end-diastolic volume; EF, ejection fraction; ESV, end-systolic volume; fRV, forward right ventricular; HR, heart rate; LV, left ventricular; RV, right ventricular; RVAD, right ventricular assist device; SV, stroke volume; TVR, tricuspid valve regurgitation (\% of SV).

${ }^{\text {a }}$ Significant difference with 8 weeks in same group.

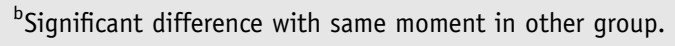


Table 3 Pressure and Flow Data

\begin{tabular}{|c|c|c|c|c|c|c|}
\hline & \multicolumn{3}{|l|}{8 weeks } & \multicolumn{3}{|l|}{16 weeks } \\
\hline & \multicolumn{3}{|c|}{ Pump group $(n=8)$} & \multicolumn{3}{|c|}{ Pump group $(n=8)$} \\
\hline & No support & Pump on & Control $(n=8)$ & No support & Pump on & Control $(n=8)$ \\
\hline HR (beats/min) & $94 \pm 20$ & $84 \pm 24^{a}$ & $95 \pm 18$ & $97 \pm 8$ & $96 \pm 8$ & $98 \pm 14$ \\
\hline Pump flow (liters/min) & $0 \pm 0$ & $2.78 \pm 0.23^{a}$ & $0 \pm 0$ & $0 \pm 0$ & $2.28 \pm 0.42^{a, b}$ & $0 \pm 0$ \\
\hline RVCO (liters/min) & $2.74 \pm 0.57$ & $0.43 \pm 0.47^{a}$ & $2.87 \pm 0.81$ & $3.44 \pm 0.88$ & $1.63 \pm 0.63^{a, b}$ & $3.25 \pm 0.77$ \\
\hline Total C0 (liters/min) & $2.74 \pm 0.57$ & $3.20 \pm 0.54$ & $2.87 \pm 0.81$ & $3.44 \pm 0.88$ & $3.91 \pm 0.83$ & $3.25 \pm 0.77$ \\
\hline $\mathrm{mABP}(\mathrm{mm} \mathrm{Hg})$ & $63.3 \pm 14.6$ & $71.8 \pm 14.1^{a}$ & $62.4 \pm 13.8$ & $60.0 \pm 12.6$ & $67.5 \pm 14.0^{a}$ & $60.6 \pm 8.8$ \\
\hline mPAP proximal $(\mathrm{mm} \mathrm{Hg})$ & $26.8 \pm 3.7$ & $32.4 \pm 4.9^{a}$ & $27.0 \pm 3.9$ & $33.8 \pm 3.0^{b}$ & $32.8 \pm 3.1$ & $26.0 \pm 6.2^{c}$ \\
\hline Systolic & $41.4 \pm 9.3$ & $39.0 \pm 10.1$ & $41.7 \pm 9.1$ & $49.0 \pm 7.2^{b}$ & $44.5 \pm 8.3$ & $37.5 \pm 8.1^{c}$ \\
\hline Diastolic & $13.0 \pm 2.0$ & $23.6 \pm 7.2^{\mathrm{a}}$ & $12.9 \pm 2.3$ & $21.1 \pm 3.4^{b}$ & $23.8 \pm 3.9^{\mathrm{a}}$ & $17.8 \pm 3.9^{b}$ \\
\hline mPAP distal $(\mathrm{mm} \mathrm{Hg})$ & $17.3 \pm 3.8$ & $20.6 \pm 6.7^{a}$ & $17.5 \pm 4.0$ & $18.3 \pm 1.5$ & $18.1 \pm 1.7$ & $16.8 \pm 2.9$ \\
\hline $\mathrm{m} \Delta \mathrm{P}$ across band $(\mathrm{mm} \mathrm{Hg})$ & $9.5 \pm 5.0$ & $11.8 \pm 6.1$ & $9.5 \pm 4.7$ & $15.5 \pm 3.6^{\mathrm{b}}$ & $14.6 \pm 2.9$ & $9.3 \pm 6.1^{c}$ \\
\hline $\mathrm{mCVP}(\mathrm{mm} \mathrm{Hg})$ & $13.6 \pm 2.4$ & $12.8 \pm 2.8^{\mathrm{a}}$ & $13.7 \pm 3.2$ & $10.6 \pm 3.0^{\mathrm{b}}$ & $8.5 \pm 3.7^{\mathrm{a}, \mathrm{b}}$ & $14.6 \pm 3.1^{b, c}$ \\
\hline $\mathrm{mLAP}(\mathrm{mm} \mathrm{Hg})$ & $6.1 \pm 2.0$ & $8.6 \pm 2.9^{a}$ & $6.2 \pm 2.2$ & $5.9 \pm 2.0$ & $5.3 \pm 2.0$ & $9.1 \pm 3.2^{b, c}$ \\
\hline RV power (W) & $0.16 \pm 0.05$ & $0.03 \pm 0.03^{a}$ & $0.17 \pm 0.05$ & $0.25 \pm 0.06^{b}$ & $0.12 \pm 0.05^{a b}$ & $0.18 \pm 0.06^{c}$ \\
\hline Total power (W) & $0.16 \pm 0.05$ & $0.22 \pm 0.06$ & $0.17 \pm 0.05$ & $0.25 \pm 0.06^{b}$ & $0.32 \pm 0.12$ & $0.18 \pm 0.06^{c}$ \\
\hline
\end{tabular}

pressures increased significantly. The resulting significantly increased $\mathrm{SW}$ and $\mathrm{RV}$ power, together with increased measured $\operatorname{RVCO}(p=0.07)$ and significantly lower CVP, illustrate RV recovery. Slightly increased $\mathrm{E}_{\mathrm{es}}$ and significantly decreased $\mathrm{V}_{0}$ indicate increased contractility, as confirmed by the significantly decreased $\mathrm{V}_{40}$. RV capacitance decreased significantly.

\section{RV function in control vs pump animals}

Sixteen weeks after PA banding, controls were compared with pump animals (Tables 2, 3, 4 and 5, and Figures 2 and
3B vs C). MRI showed increased EDV and ESV with significantly higher SV. However, forward SV and EF were significantly smaller, indicating worsening RV systolic function. Despite the calculated RVCO being significantly higher, the RVCO measured was comparable. All these findings can be attributed to the significantly further increased TVR. In contrast, TVR had almost disappeared in pump animals. Significantly higher left atrial pressures do not reflect better left atrial filling, as RVCO was comparable, but reflected increased dimensions and pressures in the adjacent right heart. CVP and EDP were both significantly higher. Further RV remodeling resulted in

Table 4 PressureVolume Loopderived Parameters

\begin{tabular}{|c|c|c|c|c|c|c|}
\hline \multirow[b]{3}{*}{ RV parameters } & \multicolumn{3}{|l|}{8 weeks } & \multicolumn{3}{|l|}{16 weeks } \\
\hline & \multicolumn{3}{|c|}{ Pump group $(n=8)$} & \multicolumn{3}{|c|}{ Pump group $(n=8)$} \\
\hline & No support & Pump on & Control $(n=8)$ & No support & Pump on & Control $(n=8)$ \\
\hline Peak RV pressure $(\mathrm{mm} \mathrm{Hg})$ & $40.4 \pm 6.5$ & $30.7 \pm 7.6^{a}$ & $42.1 \pm 8.4$ & $46.5 \pm 7.3^{b}$ & $39.4 \pm 6.9^{a, b}$ & $53.9 \pm 9.6^{b}$ \\
\hline $\mathrm{EDP}(\mathrm{mm} \mathrm{Hg})$ & $10.1 \pm 4.1$ & $7.1 \pm 3.0^{a}$ & $10.2 \pm 3.7$ & $9.8 \pm 2.2$ & $6.9 \pm 2.2^{a}$ & $13.3 \pm 3.8^{b, c}$ \\
\hline $\mathrm{ESP}(\mathrm{mm} \mathrm{Hg})$ & $39.3 \pm 6.9$ & $30.3 \pm 7.8^{a}$ & $40.0 \pm 8.2$ & $45.8 \pm 8.1^{\mathrm{b}}$ & $39.0 \pm 7.1^{\mathrm{a}, \mathrm{b}}$ & $53.1 \pm 9.4^{b}$ \\
\hline EDV $(\mathrm{ml})$ & $129.6 \pm 28.7$ & $97.5 \pm 29.8^{a}$ & $139.3 \pm 16.1$ & $104.2 \pm 20.4^{b}$ & $65.6 \pm 22.2^{a, b}$ & $145.9 \pm 23.3^{c}$ \\
\hline ESV (ml) & $92.0 \pm 25.9$ & $80.2 \pm 27.3^{a}$ & $100.0 \pm 20.3$ & $66.3 \pm 18.4^{b}$ & $43.3 \pm 23.0^{a, b}$ & $92.1 \pm 23.9^{c}$ \\
\hline SV $(\mathrm{ml})$ & $37.6 \pm 12.5$ & $17.3 \pm 5.3^{a}$ & $39.1 \pm 14.7$ & $38.0 \pm 10.1$ & $22.3 \pm 10.8^{a}$ & $53.8 \pm 18.0^{\mathrm{b}, \mathrm{c}}$ \\
\hline $\mathrm{EF}(\%)$ & $30 \pm 9$ & $19 \pm 6^{a}$ & $28 \pm 11$ & $37 \pm 10^{b}$ & $38 \pm 21^{b}$ & $37 \pm 11^{\mathrm{b}}$ \\
\hline $\mathrm{SW}(\mathrm{ml} \times \mathrm{mm} \mathrm{Hg})$ & $908 \pm 360$ & $92 \pm 65^{a}$ & $988 \pm 394$ & $1,084 \pm 281^{b}$ & $538 \pm 199^{a, b}$ & $1,609 \pm 726^{b}$ \\
\hline PVA $(\mathrm{ml} \times \mathrm{mm} \mathrm{Hg})$ & $2,110 \pm 713$ & $503 \pm 409^{a}$ & $2,521 \pm 862$ & $2,613 \pm 974$ & $1,357 \pm 714^{\mathrm{a}, \mathrm{b}}$ & $4,727 \pm 1,499^{b, c}$ \\
\hline
\end{tabular}

$E F$, ejection fraction; EDP, end-diastolic pressure; EDV, end-diastolic volume; ESP, end-systolic pressure; ESV, end-systolic volume; PVA, pressurevolume area; RV, right ventricular; SV, stroke volume; SW, stroke work.

${ }^{\text {a }}$ Significant difference vs no support at the time-point.

${ }^{\text {b}}$ Significant difference vs the same group and condition at 8 weeks.

'Significant difference vs no support in pump group at the same time-point. 
Table 5 Contractility and Compliance

\begin{tabular}{|c|c|c|c|c|}
\hline & \multicolumn{2}{|l|}{8 weeks $(n=8)$} & \multicolumn{2}{|l|}{16 weeks $(n=8)$} \\
\hline \multicolumn{5}{|l|}{ Pump on (single-beat methods) } \\
\hline \multicolumn{5}{|l|}{ Contractility } \\
\hline ESPVR $\left[E_{\mathrm{es}}(\mathrm{mm} \mathrm{Hg} / \mathrm{ml})\right]$ & \multicolumn{2}{|l|}{$1.09 \pm 0.33$} & \multicolumn{2}{|l|}{$1.27 \pm 0.97$} \\
\hline ESPVR $\left[\mathrm{V}_{0}(\mathrm{ml})\right]$ & \multicolumn{2}{|l|}{$49.5 \pm 34.6$} & \multicolumn{2}{|l|}{$0.62 \pm 32.3^{a}$} \\
\hline ESPVR $\left[\mathrm{V}_{40}(\mathrm{ml})\right]$ & \multicolumn{2}{|l|}{$90.2 \pm 32.1$} & \multicolumn{2}{|l|}{$43.7 \pm 22.5^{a}$} \\
\hline \multicolumn{5}{|l|}{ Compliance } \\
\hline \multirow[t]{2}{*}{ EDPVR $\left[\mathrm{V}_{30}(\mathrm{ml})\right]$} & \multicolumn{2}{|l|}{$126.2 \pm 33.0$} & \multicolumn{2}{|l|}{$85.2 \pm 28.3^{a}$} \\
\hline & \multicolumn{2}{|l|}{8 weeks } & \multicolumn{2}{|l|}{16 weeks } \\
\hline Pump off (pre-load reductions) & Pump group $(n=8)$ & Control group $(n=8)$ & Pump group $(n=8)$ & Control group $(n=8)$ \\
\hline \multicolumn{5}{|l|}{ Contractility } \\
\hline ESPVR $\left[\mathrm{E}_{\mathrm{es}}(\mathrm{mm} \mathrm{Hg} / \mathrm{ml})\right]$ & $0.61 \pm 0.16$ & $0.59 \pm 0.17$ & $0.69 \pm 0.20$ & $0.44 \pm 0.15^{a, b}$ \\
\hline $\operatorname{ESPVR}\left[\mathrm{V}_{0}(\mathrm{ml})\right]$ & $24.1 \pm 31.0$ & $31.7 \pm 29.8$ & $-4.6 \pm 19.1^{\mathrm{a}}$ & $-35.5 \pm 41.6^{a, b}$ \\
\hline ESPVR $\left[\mathrm{V}_{40}(\mathrm{ml})\right]$ & $93.5 \pm 28.8$ & $99.4 \pm 26.6$ & $58.2 \pm 12.6^{\mathrm{a}}$ & $55.4 \pm 33.4^{\mathrm{a}}$ \\
\hline \multicolumn{5}{|l|}{ Compliance } \\
\hline EDPVR $\left[\mathrm{V}_{30}(\mathrm{ml})\right]$ & $158.5 \pm 30.7$ & $167.4 \pm 29.3$ & $126.9 \pm 25.4^{\mathrm{a}}$ & $167.7 \pm 23.56^{b}$ \\
\hline In (EDPVR) $[\beta(\mathrm{mm} \mathrm{Hg} / \mathrm{ml})]$ & $5.84 \pm 0.16$ & $5.87 \pm 0.15$ & $5.85 \pm 0.06$ & $5.97 \pm 0.13^{a, b}$ \\
\hline $\ln \left(\right.$ EDPVR) $\left[\mathrm{V}_{0}(\mathrm{ml})\right]$ & $4.46 \pm 0.21$ & $4.54 \pm 0.23$ & $4.24 \pm 0.20^{a}$ & $4.54 \pm 0.15^{b}$ \\
\hline
\end{tabular}

EDPVR, end-diastolic pressure-volume relationship; $E_{\text {es }}$, end-systolic elastance; $E S P V R$, end-systolic pressure-volume relationship; $V_{x}$, volume intercept for a given pressure of $\times \mathrm{mm} \mathrm{Hg} ; \beta$, slope of logarithmic transformation of EDPVR.

${ }^{a}$ Significant difference vs 8 weeks before in same group.

${ }^{\text {b}}$ Significant difference vs other group at same time-point.

higher peak RV pressure and ESP compared with 8 weeks earlier, even slightly higher than in pump animals. Higher SV and ESP resulted in further increased SW and PVA. SW was higher $(p=0.077)$ and PVA significantly higher than in pump animals. Contractility also improved, as manifested by significantly decreased $\mathrm{V}_{40}$, but with significantly lower $\mathrm{E}_{\mathrm{es}}$ and $\mathrm{V}_{0}$, and $\mathrm{V}_{40}$ comparable with pump animals. Despite higher pressures and SV compared to pump animals, massive TVR prevented RVCO and PA pressures to increase. PA-pressures were even significantly lower than in pump animals. Thus, the resulting forward RV power delivered was significantly lower. In contrast to pump animals, RV capacitance remained comparable with 8 weeks earlier. The slope $(\beta)$ and $V_{0}$ of the linearized EDPVR were both significantly lower, with the significantly lower $V_{30}$ indicating significantly decreased compliance.

\section{LV function}

After 8 weeks of mechanical support, MRI analysis (Table 2) did not show significant changes in LVEDV. However, LVESV decreased significantly, resulting in significantly increased LVSV and LVEF. These parameters did not change in the control group. Consequently, LVEF became significantly higher in pump vs control animals.

\section{Histologic analysis}

Figure 4 shows the histologic differences between pump and control animals at 16 weeks. Significantly more transected myocytes illustrate decreased RV muscular hypertrophy due to RV mechanical unloading, resulting in a significantly higher Fulton index. These RVs had significantly higher percentages of fibrosis. However, as RV mass was decreased, the calculated absolute mass of RV collagen did not show a significant difference. Sub-analysis of types I and III collagen did not reveal any difference.

\section{Discussion}

In this study we have assessed the effects of prolonged low-flow RV mechanical support on an animal model of chronic RV pressure overload. The pathophysiology of RV failure in PAH has recently been discussed extensively and the RV itself has been shown to be a potential therapeutic target. $^{1,16}$

\section{Rationale for low-flow RVADs}

Pressure-independent, cardiac-specific effects of current PAH treatment are limited. ${ }^{17}$ Medical treatments that both reduce PVR and improve RV function are difficult to develop because of conflicting priorities within different cell populations of heart and lung with respect to contractility, angiogenesis and apoptosis. Another strategy is needed to specifically support RV function and reduce RV wall stress, even in the context of continuing pressure overload. ${ }^{1}$ Incentives to use low-flow RVADs are based on: (1) good results with these devices in selected cases of LV failure; (2) potential benefits for the after-load-sensitive RV; (3) avoidance of excessive increases in PA pressure; and (4) PAH survival that is linked to CO. ${ }^{9}$ 

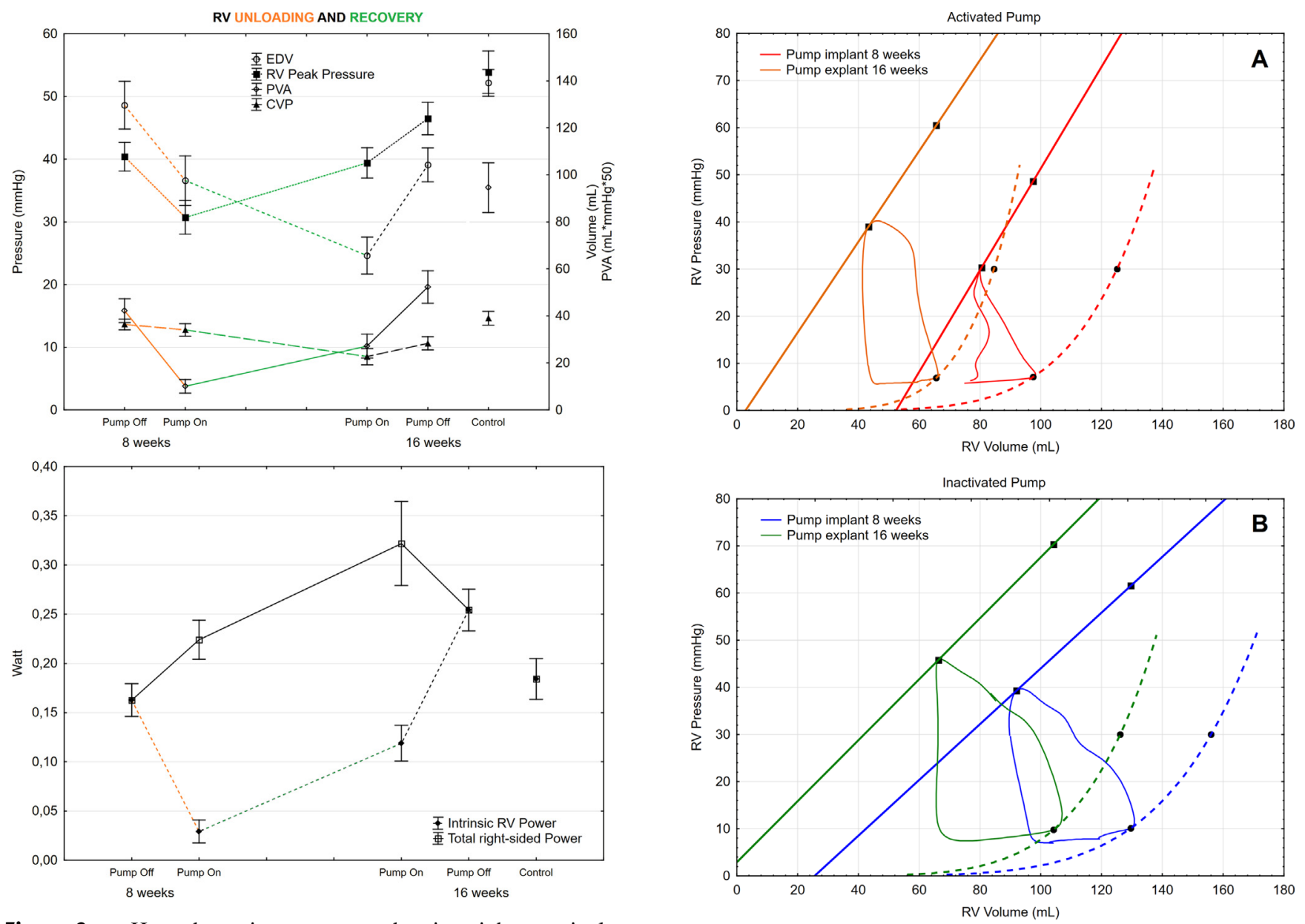

Figure 2 Hemodynamic parameters showing right ventricular unloading and recovery. $\mathrm{EDV}=$ end-diastolic volume; $\mathrm{RV}=$ right ventricle; $\mathrm{PVA}=$ pressure-volume area; $\mathrm{CVP}=$ central venous pressure.

\section{RV function}

Mechanically supporting the pressure-overloaded RV has 2 goals: improving end-organ perfusion (total $\mathrm{CO}$ and $\mathrm{ABP}$ ) and RV unloading (decreasing RV pressure, CVP, PVA and EDV) to improve coronary perfusion and prevent (or reverse) maladaptive remodeling. During support, EDPVR shifted leftward and RV recovery was manifested by improved contractility, pressure generation and SW, resulting in 3- to 4-fold increased RVCO and power. The resulting higher pressure gradients across the pumps led to pump flows evolving from full to partial support, and supported total $\mathrm{CO}$ became significantly higher than unsupported $\mathrm{CO}$ compared with 8 weeks earlier. After RVAD removal, EDPVR shifted leftward and RV recovery was manifested by almost complete disappearance of TVR and significantly improved RV pressure, SW and contractility.

At 16 weeks, control RV myocytes increased in size, causing slightly higher pressures and SW. This remodeling is maladaptive, as EDV increased further, causing TVR and oxygen consumption (PVA) to increase further, yet CO did not. The resulting significantly lower right-sided forward power in controls made RV function less efficient. Leftward shifted EDPVR and smaller EDV and RV myocytes in

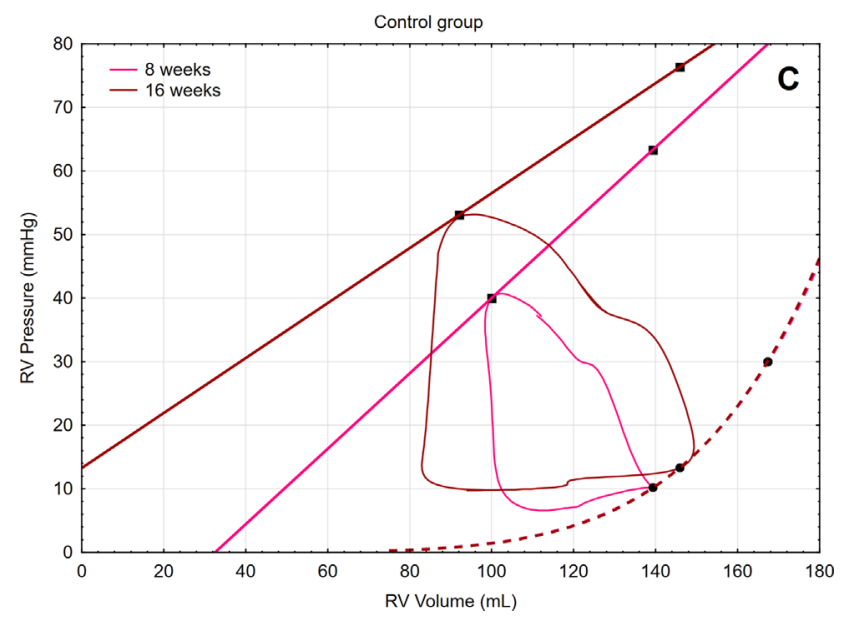

Figure 3 Pressure-volume (PV) loops. (A) PV loops of the supported RV in the pump group. (B) PV loops of the unsupported $\mathrm{RV}$ in the pump group. (C) PV loops in the control group. Squares: end-systolic point on the PV loop and maximum pressure point $\left(\mathrm{P}_{\max }\right)$, through which the end-systolic PV relation (ESPVR) is drawn; circles: end-diastolic point on the PV loop, through which the end-diastolic PV relation (EDPVR) is drawn and the point on this EDPVR at a pressure of $30 \mathrm{~mm} \mathrm{Hg}$.

pump animals reflected reverse remodeling, as seen with LV assist device (LVAD) therapy. ${ }^{18-20}$ It is unclear whether this implies true RV myocardial recovery. LV fibrosis contributes to low rates of full ventricular recovery and to high rates of recurrent remodeling, respectively, during and after 


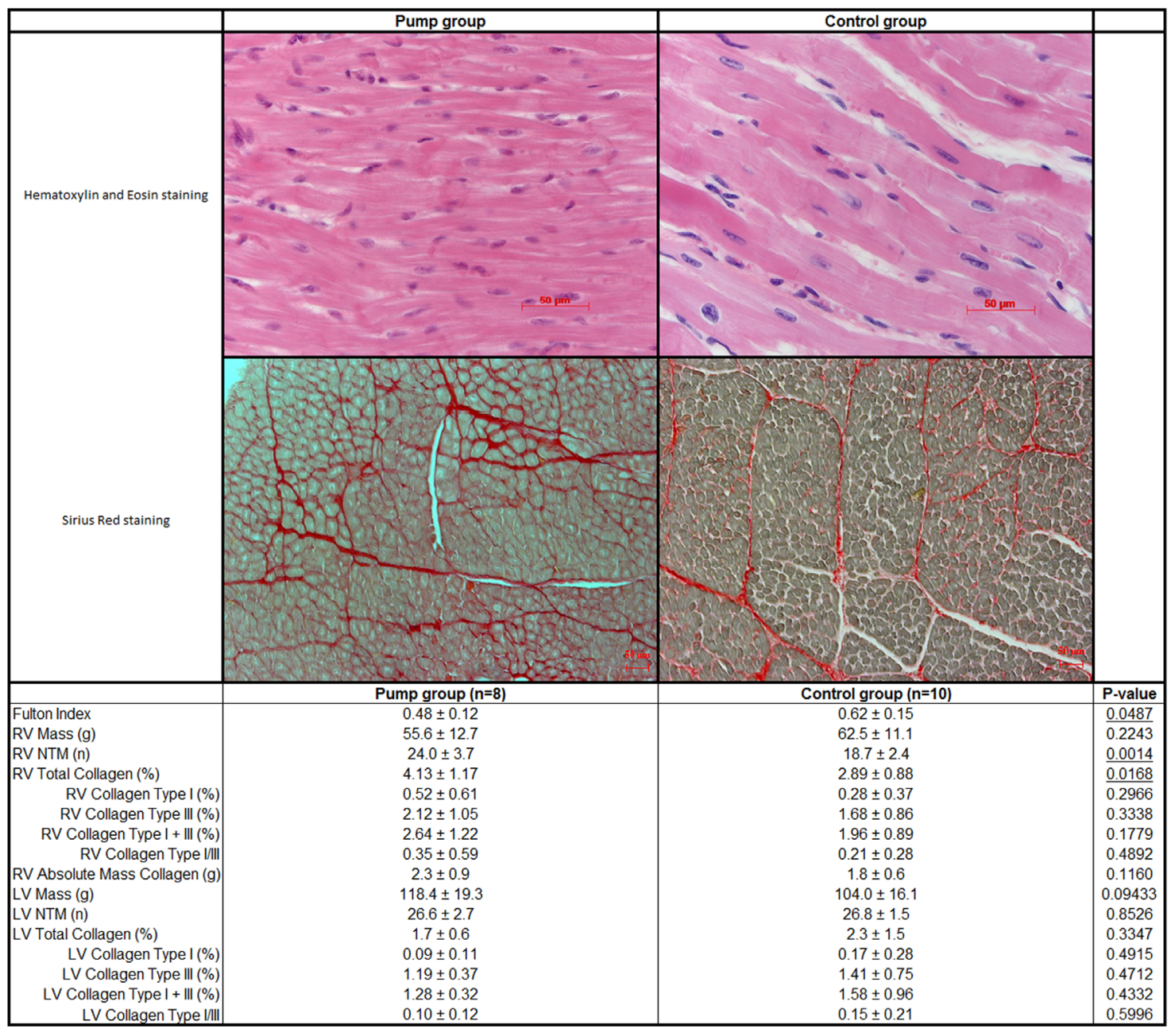

Figure 4 Histologic analysis. $\mathrm{RV}=$ right ventricle; $\mathrm{NTM}=$ number of transected myocytes; $\mathrm{LV}=$ left ventricle.

LVAD support. ${ }^{18-20}$ RV fibrosis due to pressure overload, however, is much lower and often limited to RV septal insertion points. $^{21,22}$ This may explain the generally good RV recovery after bLTx. ${ }^{23}$ The increased percentage of fibrosis made us apprehensive at first; however, as RV mass in control animals was $12 \%$ larger compared with pump animals, we calculated absolute fibrotic mass to exclude this reference trap. ${ }^{18}$ This calculation showed that the absolute amount of RV fibrosis did not differ significantly from controls. This may seem irrelevant for destination therapy. However, increased fibrosis could affect RV recovery postbLTx. In this regard, increased RV compliance in pump animals and comparable amounts of types I and III collagen and type I:III ratio, typically increased in LVAD-induced LV fibrosis, are reassuring. ${ }^{20}$ Even so, simple extrapolation of $\mathrm{LV}$ findings to the RV should be done with caution.

\section{LV function}

To prevent failure of the chronic pre-load-deprived LV after bLTx for PAH, we proposed atrial septostomy before bLTx to train the $\mathrm{LV}^{24}$ The RVAD in this study increased total CO significantly, causing increased LV filling, and could therefore have similar effects. In fact, MRI showed a tendency for lower LVESV; higher LVSV, LVCO and LV mass; and significantly higher LVEF compared with controls. It remains unclear whether these findings are due to increasing pre-load or to geometric effects of $\mathrm{RV}$ unloading, or to a combination of both.

\section{Influence on pulmonary vasculature}

RVADs for PAH were initially believed to be detrimental as they would increase PA pressures and cause lung injury. ${ }^{25}$ This seemed confirmed by an RVAD implantation that immediately resulted in supra-systemic PAH and pulmonary hemorrhage. ${ }^{26}$ However, flow rates were not reported, and diagnosis of true PAH was questioned. ${ }^{25}$ We believe these complications can be avoided by continuous low flow due to the following reasons: (1) We previously demonstrated that PA pressure and systolic after-load increases are RVAD-flow-dependent, with low flows improving hemodynamics sufficiently. ${ }^{8}$ (2) In end-stage PAH, PA pressure increases to a new "set-point" and remains fixed thereafter. Thus, decreasing CO, and not increasing PA pressure, causes PVR to increase. ${ }^{27}$ Therefore, increasing CO toward previously tolerated levels should not increase PA pressure that much and should even lower PVR. (3) Mean PA pressures increased modestly in this experiment. The characteristics of continuous-flow devices caused this to be determined mainly by increased diastolic pressures. 


\section{Hemocompatibility}

At the time of these experiments, no durable device on the market was known to deliver low flow against high after-load. The Synergy micro-pump offered promise, but it caused overly high rates of thrombosis as LVAD. Three of our pumps thrombosed; however, animals did not receive anti-coagulation. Even so, although developing durable devices for such an application will be challenging, our results indicate that it is worthwhile to engineer and test new pump designs under such conditions.

\section{Clinical application}

Rajdev et al described a PAH case complicated by cardiogenic shock that was supported by an RVAD running at 1.9 liters/min for 56 hours without evidence of pulmonary hemorrhage. ${ }^{28}$ PA pressures increased modestly, whereas CO increased significantly while PVR decreased. Recently, Rosenzweig et al reported on the first successful durable RVAD support in a non-transplant-eligible PAH patient with end-stage RV failure. ${ }^{29}$ This case coincides with our experimental findings, as mean PA pressure increased with 8 to $10 \mathrm{~mm} \mathrm{Hg}$, without a significant increase in systolic PA pressure. LV size increased. The patient clinically improved, but died from pneumonia after 16 days. Autopsy revealed no pulmonary embolism or pulmonary hemorrhage, and an RVAD free of thrombosis.

To our knowledge, we are the first to describe hemodynamic and histologic effects of prolonged RV mechanical support on the chronic pressure-overloaded RV in an experimental setting. Although further research is necessary concerning indications and correct timing, we believe these results encourage future use of this novel therapeutic strategy for PAH patients with worsening RV function.

\section{Limitations}

This study has some limitations. First, the small number of animals may impact the significance and interpretation of results. Second, our animal model is not completely clinically representative. It does not model the primary pathophysiology in the PA tree. However, to the best of our knowledge, no large PAH animal model exists that provides sufficient RV pressure overload to such an extent as PA banding. Hemodynamic maximal tolerated PA banding caused mean PA pressures to reach $67 \%$ of mean systemic pressures, distinguishing our model from the near-systemic PA pressures reached in end-stage PAH. Third, adaptive hypertrophy and dilation from fixed banding may be different from hypertrophy and dilation from progressive inflatable banding, which may better mimic chronic RV pressure overload.

\section{Conclusions}

In conclusion, prolonged continuous low-flow RV mechanical support significantly unloads the chronic pressure overloaded RV and improves CO. It modestly increases PA pressure, but mainly its diastolic component. After 8 weeks, LV function improves and RV hemodynamic recovery and reverse remodeling emerge, without a concomitant increase in fibrosis. This encourages future use of this novel therapeutic strategy for PAH patients with worsening RV function.

\section{Disclosure statement}

D.B. is consultant to the HeartWare Division of Medtronic. F.R. and B.M. received research grants from HeartWare, but the grant support was unrelated to this investigation. This study was supported by a research grant from KU Leuven, and partially supported by SensorArt (EU FP7 project). R.B.D. is recipient of a postdoctoral fellowship from the Research Foundation Flanders (FWO Vlaanderen). CircuLite provided the Synergy micro-pump. The authors thank Ingrid Van Tichelen, Stéphanie De Vleeschauwer, Michael Martin, David Célis and Jelle Verhoeven, and all the visiting students, for their valuable help during surgery and for caretaking of the animals and the handling of the pumps.

\section{References}

1. Bogaard HJ, Abe K, Vonk Noordegraaf A, et al. The right ventricle under pressure: cellular and molecular mechanisms of right-heart failure in pulmonary hypertension. Chest 2009;135:794-804.

2. McLaughlin VV, Archer SL, Badesch DB, et al. ACCF/AHA 2009 Expert Consensus Document on Pulmonary Hypertension. A Report of the American College of Cardiology Foundation Task Force on Expert Consensus Documents and the American Heart Association developed in collaboration with the American College of Chest Physicians; American Thoracic Society, Inc; and the Pulmonary Hypertension Association. Circulation 2009;119:2250-94.

3. Ritchie M, Waggoner AD, Dávila-Román VG, et al. Echocardiographic characterization of the improvement in right ventricular function in patients with severe pulmonary hypertension after singlelung transplantation. J Am Coll Cardiol 1993;22:1170-4.

4. Sandoval J, Rothman A, Pulido T. Atrial septostomy for pulmonary hypertension. Clin Chest Med 2001;22:547-60.

5. Keogh A, Mayer E, Benza RL, et al. Interventional and surgical modalities of treatment in pulmonary hypertension. J Am Coll Cardiol 2009;54(suppl):S67-77.

6. Kapur NK, Paruchuri V, Jagannathan A, et al. Mechanical circulatory support for right ventricular failure. JACC Heart Fail 2013;1:127-34.

7. Berman $\mathrm{M}$, Tsui $\mathrm{S}$, Vuylsteke $\mathrm{A}$, et al. Life-threatening right ventricular failure in pulmonary hypertension: RVAD or ECMO? J Heart Lung Transplant 2008;27:1188-9.

8. Verbelen T, Verhoeven J, Goda M, et al. Mechanical support of the pressure overloaded right ventricle: an acute feasibility study comparing low and high flow support. Am J Physiol Heart Circ Physiol 2015;309:H615-24.

9. Verbelen T, Burkhoff D, Kasama K, et al. Systolic and diastolic unloading by mechanical support of the acute vs the chronic pressure overloaded right ventricle. J Heart Lung Transplant 2017;36:457-65.

10. Dunnill MS. Quantitative methods in the study of pulmonary pathology. Thorax 1962;17:320-8.

11. Geens JH, Jacobs S, Claus P, et al. Partial mechanical circulatory support in an ovine model of post-infarction remodeling. J Heart Lung Transplant 2013;32:815-22.

12. Brimioulle S, Wauthy P, Ewalenko P, et al. Single-beat estimation of right ventricular end-systolic pressure-volume relationship. Am J Physiol Heart Circ Physiol 2003;284:H1625-30.

13. Marquardt D. An algorithm for least-squares estimation of nonlinear parameters. SIAM J Imaging Sci 1963;11:431-41. 
14. Burkhoff D, Mirsky I, Suga H. Assessment of systolic and diastolic ventricular properties via pressure-volume analysis: a guide for clinical, translational, and basic researchers. Am J Physiol Heart Circ Physiol 2005;289:H501-12.

15. Klotz S, Dickstein ML, Burkhoff D. A computational method of prediction of the end-diastolic pressure-volume relationship by single beat. Nat Protoc 2007;2:2152-8.

16. Vonk-Noordegraaf A, Haddad F, Chin KM, et al. Right heart adaptation to pulmonary arterial hypertension: physiology and pathobiology. J Am Coll Cardiol 2013;62:D22-3.

17. Handoko ML, de Man FS, Allaart CP, et al. Perspectives on novel therapeutic strategies for right heart failure in pulmonary arterial hypertension: lessons from the left heart. Eur Respir Rev 2010;19:72-82.

18. Wohlschlaeger J, Schmitz KJ, Schmid C, et al. Reverse remodeling following insertion of left ventricular assist devices (LVAD): a review of the morphological and molecular changes. Cardiovasc Res 2005:376-86.

19. Klotz S, Foronjy RF, Dickstein ML, et al. Mechanical unloading during left ventricular assist device support increases left ventricular collagen cross-linking and myocardial stiffness. Circulation 2005;112:364-74.

20. Burkhoff D, Klotz S, Mancini M. LVAD-induced reverse remodeling: basic and clinical implications for myocardial recovery. J Card Fail 2006;12:227-39.

21. McCann GP, Gan CT, Beek AM, et al. Extent of MRI delayed enhancement of myocardial mass is related to right ventricular dysfunction in pulmonary artery hypertension. AJR Am J Roentgenol 2007; 188:349-55
22. Shehata ML, Lossnitzer D, Skrok J, et al. Myocardial delayed enhancement in pulmonary hypertension: pulmonary hemodynamics, right ventricular function, and remodeling. AJR Am J Roentgenol 2011;196:87-94.

23. Kasimir MT, Seebacher G, Jaksch P, et al. Reverse cardiac remodeling in patients with primary pulmonary hypertension after isolated lung transplantation. Eur J Cardiothorac Surg 2004;26 (26):776-81.

24. Verbelen T, van Cromphaut S, Rega F, et al. Acute left ventricular failure after bilateral lung transplantation for idiopathic pulmonary arterial hypertension. J Thorac Cardiovasc Surg 2013;145:e7-9.

25. Berman M, Tsui S, Vuylsteke A, et al. Life-threatening right ventricular failure in pulmonary hypertension: RVAD or ECMO? J Heart Lung Transplant 2008;27:1188-9.

26. Gregoric ID, Chandra D, Myers TJ, et al. Extracorporeal membrane oxygenation as a bridge to emergency heart-lung transplantation in a patient with idiopathic pulmonary arterial hypertension. J Heart Lung Transplant 2008;27:466-8.

27. Voelkel MA, Wynne KM, Badesch DB, et al. Hyperuricemia in severe pulmonary hypertension. Chest 2000;117:19-24.

28. Rajdev S, Benza R, Misra V. Use of Tandem Heart as a temporary hemodynamic support option for severe pulmonary artery hypertension complicated by cardiogenic shock. J Invasive Cardiol 2007;19: E226-9.

29. Rosenzweig EB, Chicotka S, Bacchetta M. Right ventricular assist device use in ventricular failure due to pulmonary arterial hypertension: lessons learned. J Heart Lung Transplant 2016;35:1272-3. 